\title{
Temporal Naturalism-Analysis of the Paper by Lee Smolin
}

\author{
Vincent Vesterby
}

Lee Smolin has chosen to pursue a particularly difficult project. He wants to convince physicists that time is real, and that it should be an integral component of the equations of physics.

What makes this project so difficult is that no one down through the millennia has ever been able to figure out just what time is. No one has ever been able to answer the questions: What is time? Why does time exist? and Why does time have the specific qualities that it has?

The reason for this is that knowledge of the known qualities of time does not lead to knowledge of what time is. Knowledge of the qualities of time does not lead to knowledge of the basis of time in the universe.

At the time Smolin wrote this essay, he did not know what time is, and yet he was intent on convincing scientists that it is real. If no one knows what time is, the discussion about the nature of time is reduced, for the most part, to pitting speculation against speculation.

Some things are known about time. For example:

1. Time is real.

2. Everyone experiences it.

3. It is a form of change.

4. The change is unidirectional.

5. The change is progressive, with an ever increasing quantity of events that have happened but are now past.

6. The progressive change is relentless, hence the proverb "Time and tide wait for no man."

7. Actions in the present can influence what will happen in the future, but actions in the present cannot influence what has already happened and is now past.

But if someone asks "Why is time a form of change?" or "Why is it that actions in the present cannot influence the past?" all answers can be nothing more than speculation. The trouble with speculation is that anything can be said - any unrealistic notion or groundless idea. And that is what has happened with the discussion about the nature of time. The accumulation of speculation about time has been growing for thousands of years.

That is what Smolin is up against. And yet he does not have much more than speculation himself. He advocates a few of the known qualities of time, for example ". . . an embrace of the reality of time ..." (Smolin 2013 p. 3) and the "... posit that the past influences the present and future, but the reverse is not true; no event in the past can be affected by anything that happens in its future." (p. 8) The use of the terms, embrace and posit, 
indicate that Smolin does not know why time is real or why the relations of past, present, and future are what they are.

Smolin also states, "Temporal naturalism has no need to speak of the rate of flow of time." (p. 36) This is true. But because he does not know what time is - the basis of time in the universe-his reasons why the rate of flow of time is not an issue are ad hoc.

There is a paper at OSF Preprints that explains why time is not a flow. It was written in response to comments by Paul Davies about why time is not a flow. Davies was correct in his judgement that time does not flow, but, like Smolin, he gave the wrong reasons. (Vesterby 2019c)

In his paper, Smolin promotes temporal naturalism, the reality of time, by contrasting it with timeless naturalism, the block universe view of time. It is unfortunate that Smolin felt it necessary to oppose the block universe idea. A great deal of his essay is preoccupied with this unrealistic, unnatural, fiction.

Timelessness in any form has no reality in the universe. It is just a concept, an ideawhich is not the thing itself. The trouble with working only in the realm of concepts is that anything can be said about anything. There is no ground truth-no reality check.

Concepts are mental tools used by the mind to help achieve understanding of the real world, the universe. When the subject of investigation is a component or feature of the universe, it is critical to focus the attention on the reality-referents of the concepts. Dealing with the reality-referents of the concepts by way of observation puts limits on the meanings of the concepts. The basic procedure is to let the reality-referents dictate through the process of observation the content and meaning of the concepts. Let reality dictate, through the process of observation, the mind's understanding of reality.

It would have been better in his essay for Smolin to focus on time itself. It is easy to observe time - all physical processes, all changes, take time to occur. Anyone can watch physical processes taking time to occur. There is no need to contrast the understanding of the reality of time with the notions of an unreal air-castle. By focusing on time, by focusing on its evident, its observable, qualities, the reality of time becomes established on its own merits. (Vesterby 2019a, 2019b)

Unfortunately, Smolin's essay contains at least four main themes that inhibit the rational objective observation of time itself.

1. Motives that are beyond the issues at hand.

2. Giving credit where credit is not due.

3. Relationalism.

4. Preoccupation with hypothesis and theory.

5. Smolin believes that there is no scientific method. 


\section{Ulterior Motives}

Smolin wants time to be real because he believes it is critical for the evolution of the laws of science and for the existence of qualia. These are ulterior motives for the reality of time, not in the sense of covert motives, but in the sense of motives that are beyond the issue at hand - whether or not time is real. The evolution of laws and the reality of qualia are not factors relevant to the question concerning the reality of time. The problem with ulterior motives is that they can cloud the judgement. They can influence a researcher to draw conclusions that support the ulterior motive, but that are not exactly accurate about what is observed.

Some ulterior motives are just distractions from the necessity of focusing on the actual topic of investigation - time. The evolution of laws and the reality of qualia are ulterior motives of this type. Discussion of these two distractions does not lead to an understanding of time or its intrinsic qualities.

\section{Giving Credit Where Credit Is Not Due}

There are two outstanding cases of giving undue significance to topics in Smolin's essay - timeless naturalism and panpsychism. These are both devoid of observable qualities. Because timeless naturalism is unreal, it is not natural. When trying to achieve an accurate understanding of the intrinsic nature of time and its qualities, it is not appropriate procedure to compare and contrast it with something that has no basis in reality.

Panpsychism is entirely anthropomorphic. It is a case of extreme animism. Because panpsychism is false, it cannot contribute anything of value to the understanding of time or qualia. Both timeless naturalism and panpsychism are unworthy of discussion and distract from the project of convincing physicists that time is real and should have a realistic place in the equations of physics.

\section{Relationalism}

Relationalism is a case of putting the cart before the horse. In the real world, in the universe, relations occur between things that exist. Relations occur between the components of situations - if there are no components, there are no relations. The existence of the components is primary - the existence of the relations is secondary.

Thus, something that exists is not properly identified by its relations to other things that exist, but rather by what it is intrinsically - by its intrinsic-identity. The intrinsic-identity of something that exists is not based on its location, it is not based on the distance and direction relations with other locations, or by what exists at those locations. Nor is it properly identified by its interactions with other things that exist. The existence of components is primary - the existence of interactions between components is secondary.

Everything that exists, everything that is real, has its own intrinsic-identity based on its existence. The identity is based foundationally on the mode-of-being, the manner in which it exists - immaterial like space and time, or substantial like matter. 
If substantial, the intrinsic-identity is then based on what it is. For example, is it an elementary particle, or is it an atom composed of elementary particles, or a molecule composed of atoms? Material-reality is hierarchically organized, up from these foundational levels, with each emergent higher level composed of components of the lower levels. The identity of something is based on the level, for example the level of planets or the level of solar systems, the level of living cells or the level of a whole organism, the level of pieces like axels and steering wheels or the level of a car.

The identity of something is also based on what it is at a particular level, for example, at the level of fruit, is it an apple, an orange, or a banana. What it is at a its hierarchic level is based on the intrinsic pattern-of-organization, for example, are the seeds on the inside as with a watermelon or on the outside as with a strawberry.

Therefore, instead of bringing in relationalism, which is inappropriate for the identification of the nature of time, Smolin should have focused on the known qualities of time to convince physicists of the reality of time, for example the relentless change of time, its universality, and its unidirectionality, and so on.

\section{Preoccupation with Hypothesis and Theory.}

Smolin's essay has 39 pages of text. Terms relating to hypothesis and theory are used 83 times, which is slightly over two times per page. The problem is that hypothesis and theory are speculation - not facts, not knowledge, not understanding.

Knowledge is the goal of science. Knowledge about the physical nature of the universe is supposed to be the goal of physics. Theory, speculative guesswork, is not the goal of science in general or of physics.

Because the essay focuses on ulterior motives, speculations, relationalism, and a preoccupation with hypothesis and theory, there is very little mention of facts, knowledge, or understanding relating to time itself. Nobody is going to be convinced of the reality of time or the importance of time for making the equations of physics more realistic by all this discussion of extraneous issues and unrealistic speculations.

\section{No Scientific Method}

Smolin says, "... naturalists also hold that science is the most reliable route to knowledge about nature." (p. 4) He then says, "Most importantly, I need to emphasize that ... there is no scientific method ..." (p. 7), thereby making it clear that he does not know what science is. Smolin does not know why science is the most reliable route to knowledge.

The problem here is that it is specifically the scientific method that sets science apartthat makes science "the most reliable route to knowledge about nature." The basic rule of science is allow the intrinsic nature of reality to dictate to the mind the mind's knowledge and understanding of reality — reality being that which exists, the universe. The purpose of science is to provide accurate knowledge and understanding of the universe and that 
which exists within it. This goal of accurate knowledge is achieved through the application of the scientific method.

The method is based on careful observation. That is the purpose of experiments - not to prove or disprove hypotheses or theories - but simply to provide careful, accurate observations.

Foundationally, science is a procedure, a method, for obtaining accurate knowledge. The core procedure has three basic stages. This set of three stages is repeated as many times as necessary to acquire the target knowledge. In the first stage, observations are made of the subject of interest. The observations should be of a repeatable nature, that is, it should be possible to make the same observation two or more times. When the target of observation is a onetime event, and repeated observations are not possible, the observations should be of a kind that different observers can make the same observation. All observations should be checked by being made two, or preferably several times. In the second stage, the knowledge acquired through observation is integrated into an organized body of knowledge. In the third stage, the organized knowledge is examined to reveal gaps in that knowledge, that is, to reveal what is not yet known.

In the next repetition of this three-stage process, the observations focus on the gaps. The new knowledge resulting from this second round of observations is integrated into the knowledge acquired from the first observations. The new body of knowledge is examined for gaps, and the process is repeated. In this manner, science accumulates knowledge.

When making further observations to fill in gaps, it is often sufficient to focus more sharply on the area of the gap, to examine the area of the gap in more detail than was done previously. When this focused observation does not yield sufficient knowledge to fill the gap, a guess is made about what might be the nature of the knowledge that would fill the gap. This guess, this speculation, is called a hypothesis, and is used to focus the investigation on a particular possibility. The validity of the hypothesis is checked through further observation, tests, and experiments. This use of hypotheses is repeated until the solution for the gap is found.

Not just any speculation can serve as a hypothesis. First, the hypothesis must be relevant to the question at hand - relevant to the gap the hypothesis is intended to help fill in. Second, the hypothesis must be checkable. It must be of a nature such that its validity can be tested.

There are two times in the progress of scientific investigation where speculation is used to help find the knowledge that fills in what is not yet known. The first is hypothetical speculation. The second is theoretical speculation. Hypotheses are used for relatively small scale gaps, small scale issues, while theories are used for large scale knowledge issues.

The scientific process leads to massive accumulation of facts - the accumulation of large bodies of knowledge. Questions arise about how all these facts relate to one another. 
These questions represent broad, large scale gaps in understanding the accumulated knowledge.

When these broad questions are not answered by simply looking more closely, a higher level stage of speculation is used to give direction to further investigation. These higher level speculations are called theories. They consist of the accumulated body of knowledge plus the broad scale speculation that is intended to give coherence, integration, or meaning to the large body of knowledge. As with hypothesis, theory also must be relevant to the question at hand and be checkable. The new body of knowledge is examined for gaps, and the process is repeated. In this manner, science accumulates broad scale integration of knowledge.

The accumulated knowledge that results from this iterative procedure is scientific knowledge. The speculations of hypothesis and theory are not included in scientific knowledge. Hypotheses and theories do not constitute part of scientific knowledge.

A scientific theory is not science - it is just a guess, a speculation, about what should be checked next. A scientific theory is nothing more than a conceptual tool to be use at a particular stage of the multistage process of the scientific method.

\section{Focusing on Time}

To convince physicists of the reality of time it is best to focus on time itself, to focus on the known qualities of time, which can be observed. While observing the intrinsic qualities of time can lead to the understanding that time is real, knowledge of the qualities of time and knowledge that time is real does not lead to knowledge of what time is, or why it is real, or why it has the specific qualities it has. Identification of the basis of time in the universe would provide that knowledge, and make convincing others of the reality of time much easier.

\section{REFERENCES}

Smolin, Lee. 2013. Temporal Naturalism. arXiv:1310.8539v1

Vesterby, Vincent. 2019a. "The Basis of Time in the Universe." OSF Preprints. July 24. osf.io/q4ntk. https://osf.io/q4ntk

Vesterby, Vincent. 2019b. "The Identification of the Intrinsic Nature of Time." OSF Preprints. July 26. osf.io/gqxjy. https://osf.io/gqxjy

Vesterby, Vincent. 2019c. "Paul Davies and Why Time Is Not a Flow." OSF Preprints. July 27. osf.io/bh23c. https://osf.io/bh23c

Date of original version of "Temporal Naturalism-Analysis of the Paper by Lee Smolin"-2017. 